\title{
Novel Feature of Infection in Diabetic Compromized Hosts
}

It has long been recognized that diabetic patients are susceptible to infections because of various types of deficits in the host defense mechanism (1). In addition to tissue malnutrition, dehydration, vascular insufficiency or autonomic neuropathy, it is well known that chemotaxis and phagocytosis of polymorphonuclear leukocytes are decreased in the poorly controlled diabetic with dehydration $(2,3)$. Humoral factors, such as defective, inadequate gammaglobulin and distorted formation of complement and antibody depend on the type of organism and are considered to be different in each individual according to the type of diabetes (4).

In recent years, the deficiency to produce free radicals from superoxides and hydrogen peroxide provided through hexose1-phosphate pathway is attributed to a defective bacteriocidal mechanism in polymorphonuclear cells (5). As excess utilization of NADPH because of accelerated polyol pathway hyperglycemia is results in depletion of formation of superoxide (6). Although, in general, production of free-radicals is thought to be increased on glycation, NADPH is exceedingly consumed in the cells. However, the direct effect of hyperglycemia in infection has not been proven to be the cause of higher risks of infection because there is a lot of difficulty in translating complexed interaction between hyperglycemia and biological factors in a certain experimental system. Severe metabolic derangement with hyperglycemia accompanied by ketoacidosis and dehydration, common to acute complication, is considered to be the clinical manifestation accompanied by infection, and this may play a role in the further exacerbation as to induce DIC and acute renal failure.

See also p 810 .

Yoshioka $\mathrm{K}$, et al reported a novel case of clavicular osteomyelitis (7). The patient was a 53-year-old male who suffered from diabetes for 12 years with known diabetic nephropathy and retinopathy. This case suggests that osteomyelitis in a poorly controlled diabetic patient with advanced diabetic complications could occur at any site of the body, although clavicular osteomyelitis is rarely reported.

Most osteomyelitis cases are caused by a hematogenous route. However, in this case, repeated cultures from blood and synovial fluid failed to demonstrate a causative organism despite the lack of no previous administration of antibiotics. According to his past history, however, since pyogenic arthritis of the knee at about one year prior to this episode is documented, he appears to have a focus with asymptomatic relief. The local findings of ${ }^{67}$ gallium scintigram and magnetic resonance imaging (MRI) as well as computed tomography (CT) were the clues to establish the diagnosis. He survived the difficult condition of ketoacidosis, DIC and renal failure accompanied by upper-GI bleeding. He was treated with meticulous correction of metabolism, conservative antibiotics, and hemodialysis.

In diabetic patients with long-term complications, the clinical features of acute infection tend to be obscured with insidious progression. Such patients often develop an unexpected and unbelievable clinical pictures; soft tissue infection advances out of a painless foot ulcer; scrious upper urinary tract infections such as perirenal abscess or papillary necrosis are closely related to asymptomatic atonic bladder; rhinocerebral mucormycosis with ketoacidosis suddenly occurs in patients whose glycemic control is thought to be relatively good; an elderly patient developed periodontal abscess after a tooth extraction followed by failure of hydration (8). Therefore, it is very important to detect the site of infection by various methods if a patient has any single sign of an infectious disease. Because of diabetic neuropathy and the deficit of biological tissue response, a patient rarely complains of pain and as is often seen in the chronic stage of infection, lacks of local findings such as active swelling and even systemic leukocytosis. $\mathrm{HbA}_{1 \mathrm{c}}$ remains relatively low because of anemia possibly due to long standing infection and nephropathy. Moreover, the body temperature does not elevate when ketoacidosis advances.

The mortality due to diabetic ketoacidosis has remarkably decreased after the start of insulin and the mortality due to infectious diseases also decreased with development of antibiotics. However, the trend of morbidity of infectious diseases like urinary tract infection, infections related to diabetic foot lesions, and tuberculosis in diabetic patients, are not dramatically decreased in Japan (9). Again, adequate glycemic control in daily diabetes care together with patient education must be emphasized.

Kempei Matsuoka, MD Saiseikai Diabetes Center, 11-4, Mita, Minato-ku, Tokyo 108

\section{References}

1) Sentochnik DE, Epiopoulos GM. Infection and diabetes, in: Joslin's Diabetes Mellitus, CR Kahn, GC Wier, Eds. Lea \& Febiger, Philadelphia, 1994, Chapter 47, p. 867.

2) Lederman MM, Rodman HM, Schacter BZ, et al. Antibody response to pneumococcus polysaccharides in insulin-dependent diabetes mellitus. Diabetes Care 5: 36, 1982.

3) Nabhigi M, Smith RP, Baltch AL, et al. The effect of diabetes mellitus on chemotactic and bacterial activity of human polymorphonuclear leukocytes. Diabetes Res Clin Pract 4: 27, 1987.

4) Charlesworth JA, Timmermans V, Golding J. Complement system in Type 1 (insulin-dependent) diabetes. Diabetologia 30: 372, 1987. 
5) Shah SV, Wallin JD, Eilcn SD. Chemiluminescence and superoxide anion production by leukocytes from diabetic patients. J Clin Endocrinol Metab 57: 402, 1983.

6) Wilson MR, Tomlinson DR, Reaves WG. Neutrophil sorbitol production impairs oxidative killing in diabetes. Diabetic Med 4: 37, 1987.

7) Yoshioka K, Nagata H, Nakamura Y, et al. Pyogenic clavicular osteomyelitis associated with disseminated intravascular coagulation and acute renal failure in a patient with non-insulin-dependent diabetes mellitus. Intern Med 36: 810, 1997.

8) Atsumi Y, Hosokawa K, Matsuoka K. A case of diabetic gangrene and serious periodontal infection. Gendai Iryo 24: 3641, 1992.

9) Sakamoto N, Hotta N, et al. The cause of death in Japanese diabetics based on survey results among 11,648 diabetics during 1981-1990. J Jpn Diab Soc 37: 767, 1994. 